\title{
SYNTHESIS OF NOVEL SPIROTHIOBARBITURIC ACID DERIVATIVES VIA REGIOSELECTIVE MICHAEL ADDITION
}

\author{
Madhukar S Chande* and Vijay Suryanarayan \\ Department of Chemistry, The Institute of Science, 15, Madam Cama Road, Mumbai 400032, India \\ E-mail: chandems@vsnl.com,vijay.suryanarayan@gmail.com
}

\begin{abstract}
The paper describes regioselective Michael addition of 1,3-diaryl thiobarbituric acid with acceptors like methyl acrylate and acrylonitrile. The Michael adducts thus obtained were utilized as key intermediates to synthesize novel spirocyclohexanone derivatives.
\end{abstract}

\section{Introduction}

Michael addition is one of the oldest eminent reactions involving the formation of a carbon-carbon bond and has found extensive applications in organic synthesis. The reaction deals with the addition of compounds containing active methylene or methine group to activated $\pi$ systems under basic reaction conditions. This reaction has gained considerable interest with the use of organometallics ${ }^{1-4}$ and ionic liquids 5 as catalysts since it leads to products with high enantioselectivity.

In the recent past our group has been actively exploring the Michael addition reaction on various heterocyclic compounds bearing active methine ${ }^{6}$ and methylene ${ }^{7-9}$ groups. Though mono addition in compounds containing active methylene group is common, we observed that diadducts can be regioselectively obtained as pure compounds under specific reaction conditions. This unveiled new pathways to the synthesis of spirocompounds via Michael addition. This observation, coupled with the fact that thiobarbituric acids are often associated with important biological activities, prompted us to extend the scope of this methodology to synthesize novel spirothiobarbituric acid derivatives.

\section{Results and Discussions}

The 1,3-diarylthiobarbituric acids 1 were synthesized by refluxing a mixture of appropriate symmetrical thiocarbamide and malonic acid in acetyl chloride ${ }^{10}$.

The reactive methylene group at C-5 position of 1,3-diarylthiobarbituric acid makes it an active Michael donor. Thus, in presence of sodamide in DMF at ambient temperature, two moles of methyl acrylate 2 readily adds regioselectively to one mole of 1,3-diarylthiobarbituric acid 1 to furnish the diadduct 3 . The progress of the reaction was monitored by TLC. The reaction was clean without the formation of any major side products.

The Michael diadducts can now function as key intermediates to the synthesis of spirocompounds. Thus, compound 3 on treatment with base as sodium methoxide readily underwent intramolecular Dieckman condensation to 4. Acid hydrolysis of ester group in 4 with decarboxylation furnished the spirocyclohexanone derivative 5 (Scheme-1). 
To establish the generality of the reaction, it was further extended to another Michael acceptor, viz. acrylonitrile 6. Under similar reaction conditions, as anticipated, acrylonitrile 6 also undergoes double Michael addition, but is followed by insitu intramolecular Thorpe-Ziegler cyclization furnishing 7 . The hydrolysis of the nitrile and imino functional group of 7 under acidic conditions is followed by decarboxylation to furnish the cyclohexanone derivative 5 (Scheme-1). Thus an alternate synthetic route for the synthesis of 5 was achieved. The structure of 5 obtained by both the routes was confirmed unambiguously by mmp (mixed melting point), co-tlc and co-IR.<smiles>[R]c1ccc(N2C(=O)C(C)C(=O)N(c3ccc([R])cc3)C2=S)cc1</smiles><smiles></smiles>

$\left[\begin{array}{l}\mathrm{R} \\ \end{array}\right.$<smiles>[R]c1ccc(N2C(=O)C(CCC#N)(CCCC#N)C(=O)N(c3ccc([R])cc3)C2=S)cc1</smiles><smiles>[R]C(=O)OC(=O)C=CC(=O)NN</smiles><smiles>Cc1ccc(C=O)cc1</smiles><smiles>COC(=O)C1CCC(CO)(CO)CCC(=O)O1</smiles><smiles>[R]c1ccc(N=C=O)cc1</smiles><smiles>[R]c1ccc(N2C(=O)C3(CCC(=O)C(C(=O)OC)C3)C(=O)N(c3ccc([R])cc3)C2=S)cc1</smiles>

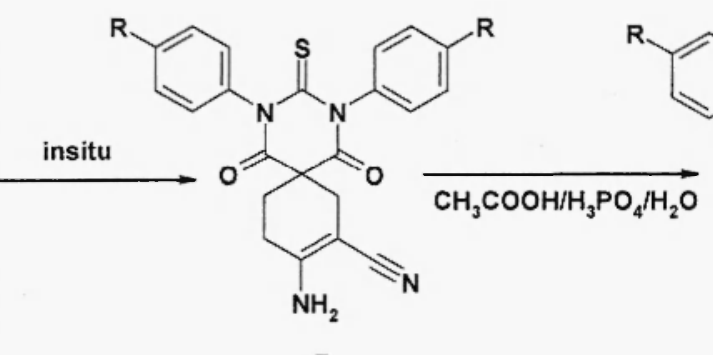

7<smiles>[R]c1ccc(N2C(=O)C3(CCC(=O)CC3)C(=O)N(c3ccc(C)cc3)C2=S)cc1</smiles>

5

Scheme-1

\section{Conclusions}

Thus, in conclusion, we report a mild and efficient method to the synthesis of novel spirocyclohexyl derivatives containing thiobarbituric acid moiety. Sodamide in DMF at ambient temperature seems an ideal condition which regioselectively leads to the formation of the diadduct. The methodology discussed in this communication is useful from a synthetic point of view and provides an easy approach to the synthesis of rather inaccessible spirocyclohexanone derivatives.

\section{Experimental Section}

Melting points reported in this paper were taken in open capillaries and are uncorrected. TLC was performed on silica gel (Acme). IR spectra were recorded on Perkin -Elmer 257-FTIR 1600 Series spectrometer by dissolving the compounds in chloroform (HPLC grade) as solvent. NMR's were recorded on a Bruker AMX 500 Series $(500 \mathrm{MHz})$ spectrometer using $\mathrm{CDCl}_{3}$ as deuterated solvent. 
Synthesis of 5,5-bis[2-(methoxycarbonyl)ethyl])-1,3-diaryl-2-thiobarbituric acids (3).

To a stirred solution of sodamide $(0.02 \mathrm{~mol})$ in DMF $(25 \mathrm{ml})$ was added 1,3-diarylthiobarbituric acid $(0.01 \mathrm{~mol})$ at $28-30^{\circ} \mathrm{C}$. This was followed by the addition of methyl acrylate $(0.02 \mathrm{~mol})$. The reaction mixture was stirred at room temperature for $4 \mathrm{hrs}$. It was then poured onto crushed ice and acidified to $\mathrm{pH}$ 2-3 with conc $\mathrm{HCl}$. The product which separated was filtered, washed well with water and crystallized with benzene: pet.ether (50:50). 3a: $\mathrm{R}=-\mathrm{CH}_{3}(94 \%)$, M.P $150^{\circ}$; IR (cm $\left.{ }^{-1}\right) 3021,2925(\mathrm{C}-$ Hstr.) $1738\left(\mathrm{C}=\mathrm{O}\right.$; ester), $1696\left(\mathrm{C}=\mathrm{O}\right.$; amide), $1214(\mathrm{C}=\mathrm{S}) .{ }^{1} \mathrm{H}-\mathrm{NMR}(\mathrm{ppm}): \partial$ 1.982-2.346(m, 8H), $2.418(\mathrm{~s}, 6 \mathrm{H}), 3.643(\mathrm{~s}, 3 \mathrm{H}), 3.843(\mathrm{~s}, 3 \mathrm{H}), 7.282-7.457(\mathrm{~m}, 8 \mathrm{H})$. Elemental Analysis (Obs/Cal) N; 5.59\% (5.64\%). 3b: R=H (93\%); M.P 192 ${ }^{\circ} \mathrm{C}$; IR ( $\left.\mathrm{cm}^{-1}\right)$ 3019, 2926 (C-H str.), 1736 (-C=O, ester), 1696 (C=O; amide), 1215 (C=S); ${ }^{1} \mathrm{H}-\mathrm{NMR}(\mathrm{ppm}): 2$ 2.023-2.338 (m, 8H), $3.647(\mathrm{~s}, 3 \mathrm{H}), 3.791(\mathrm{~s}, 3 \mathrm{H})$, 7.224-7.512 (m,10H) Elemental Analysis (Obs/Cal) N; 5.96\% (5.98\%).

Synthesis of 2,4 -diaza -1,5,9 -trioxo-10 - carbmethoxy - 3 - thio - 2,4 - diaryl - spiro[5.5] undecane(4) : 5,5-Bis[2-(methoxycarbonyl)ethyl])-1,3-diaryl-2-thiobarbituric acids (3, 0.01 mol) was added to a freshly prepared solution of sodium methoxide $(0.01 \mathrm{~mol}, 0.23 \mathrm{gms}$ in $25 \mathrm{ml}$ methanol). The reaction mixture was stirred at room temperature for $5 \mathrm{hrs}$. It was then cooled and poured onto crushed ice and acidified with conc. $\mathrm{HCl}$ to $\mathrm{pH}$ 2-3. The product was filtered, washed well with water and crystallized with ethanol. 4a $\mathrm{R}=-\mathrm{CH}_{3}(83 \%)$ : M.P $120^{\circ} \mathrm{C}$; IR $\left(\mathrm{cm}^{-1}\right): 3001(\mathrm{C}-\mathrm{H} \mathrm{str}), 1721(\mathrm{C}=\mathrm{O}$; ester), 1708 ( $\mathrm{C}=\mathrm{O}$; amide), 1208 (C=S). ${ }^{\mathrm{L}} \mathrm{H}-\mathrm{NMR}$ (ppm): $\partial$ 1.954-2.292 (m, 6H), $2.406(\mathrm{~s}, 6 \mathrm{H}), 4.098$ (s, 3H), 4.201 (br, 1H), 7.122-7.375 (m, 8H) ; Elemental Analysis (Obs/Cal) N; 5.98\% (6.03\%). 4b $\mathrm{R}=\mathrm{H}(84 \%) ; \mathrm{M} . \mathrm{P} 81^{0} \mathrm{C}$; IR ( $\left.\mathrm{cm}^{-1}, \mathrm{CHCl}_{3}\right) 3010$ (C-H str), 1725 (C=O;ester), 1708 (C=O;amide), 1208 $(\mathrm{C}=\mathrm{S}) .{ }^{\mathrm{l}} \mathrm{H}-\mathrm{NMR}(\mathrm{ppm}): \partial$ 1.856-2.254 (m, 6H), $3.895(\mathrm{~s}, 3 \mathrm{H}), 4.192(\mathrm{br}, 1 \mathrm{H}), 7.218-7.487(\mathrm{~m}, 10 \mathrm{H})$; Elemental Analysis (Obs/Cal) N; 6.36\% (6.42\%).

Synthesis of 9-amino-2,4-diaza-1,5-dioxo-10-cyano-3-thio-2,4-diaryl-spiro[5.5]dec-9,10-diene (7) : To a stirred solution of sodamide $(0.02 \mathrm{~mol})$ in DMF ( $25 \mathrm{ml})$ was added 1,3 diarylthiobarbituric acid $(0.01 \mathrm{~mol})$ at $28-30^{\circ} \mathrm{C}$. This was followed by the addition of acrylonitrile $(0.02 \mathrm{~mol})$. The reaction mixture was stirred at room temperature and monitored by TLC for completion of reaction. It was then poured onto crushed ice and acidified to $\mathrm{pH} \mathrm{2-3} \mathrm{with} \mathrm{conc} \mathrm{HCl}$. The product which separated was filtered, washed well with water and crystallized with $50 \%$ ethanol. 7a $\mathrm{R}=-\mathrm{CH}_{3}(90 \%)$, M.P $138^{\circ} \mathrm{C}(\mathrm{d}), \mathrm{IR}\left(\mathrm{cm}^{-1}, \mathrm{CHCl}_{3}\right) ; 3342\left(\mathrm{br},-\mathrm{NH}_{2}\right), 3003(\mathrm{C}-\mathrm{H} \mathrm{str}), 2245(\mathrm{C} \equiv \mathrm{N}), 1675(\mathrm{C}=\mathrm{O}), 1216(\mathrm{C}=\mathrm{S})$. ${ }^{\mathrm{l}} \mathrm{H}-\mathrm{NMR}$ (ppm): $\partial$ 2.252-2.381 (m, 6H), 2.402 (s, 6H), 4.326 (br, 2H, $\mathrm{D}_{2} \mathrm{O}$ exchangeable), 7.052 7.336 (m, 8H); Elemental Analysis (Obs/Calc) N; 12.99\%( 13.01\%). 7b R=H (91\%); M.P 192 C; IR $\left(\mathrm{cm}^{-\mathrm{i}}\right.$. $\mathrm{CHCl3}$ ); 3345 (br, -NH 2$), 3012$ (C-H str.), $2241(\mathrm{C} \equiv \mathrm{N}), 1676(\mathrm{C}=\mathrm{O}), 1214(\mathrm{C}=\mathrm{S}) .{ }^{1} \mathrm{H}-\mathrm{NMR}$ (ppm): $\partial 2.248-2.376(\mathrm{~m}, 6 \mathrm{H}), 4.324$ (br, 2H, $\mathrm{D}_{2} \mathrm{O}$ exchangeable), 7.031 - $7.433(\mathrm{~m}, 10 \mathrm{H})$ Elemental Analysis (Obs/Cal) N ; 13.89\% (13.92\%). 
Synthesis of 2,4-diaza-1,5,9-trioxo-3-thio-2,4-diaryl-spiro [5.5] undecane (5):

(a) from 4: 2,4-Diaza-1,5,9-trioxo-10-carbmethoxy-3-thio-2,4-diaryl-spiro[5.5] undecane (4, $0.005 \mathrm{~mol})$ was dissolved in a mixture of acetic acid $(10 \mathrm{ml})$ and water $(2 \mathrm{ml})$. To this was then added conc. $\mathrm{HCl}(20 \mathrm{ml})$ and the reaction mixture was heated on a heating mantle for $4 \mathrm{hrs}$. On cooling, a pale brown coloured product separated which was filtered, washed well with water, and crystallized from $50 \%$ ethanol. 5a R=- $\mathrm{CH}_{3}(83 \%)$, M.P $230^{\circ} \mathrm{C}$ (d), IR (cm $\left.{ }^{-\mathrm{i}} . \mathrm{CHCl}_{3}\right) 3032 \& 3000$ (C-H str.), 1695 (broad, $-\mathrm{C}=\mathrm{O}$ ), $1222(\mathrm{C}=\mathrm{S}) .{ }^{1} \mathrm{H}-\mathrm{NMR}(\mathrm{ppm}): \partial 2.254-2.492(\mathrm{~m}, 8 \mathrm{H}), 2.401(\mathrm{~s}, 6 \mathrm{H}), 7.182(\mathrm{~d}, 4 \mathrm{H})$, $7.272(\mathrm{~d}, 4 \mathrm{H})$. Elemental Analysis (Obs/Calc) N; 6.83\% (6.89\%). 5b R $=\mathrm{H}(86 \%), \mathrm{M} . \mathrm{P}>250^{\circ} \mathrm{C}$, IR $\left(\mathrm{cm}^{-1}, \mathrm{CHCl}_{3}\right) 3042 \& 2995$ (C-H str.), 1698 (br, $\left.-\mathrm{C}=\mathrm{O}\right), 1226$ (C=S). ${ }^{1} \mathrm{H}-\mathrm{NMR}(\mathrm{ppm}): 2$ 2.252-2.483 $(\mathrm{m}, 8 \mathrm{H}), 7.125-7.298(\mathrm{~m}, 10 \mathrm{H})$; Elemental Analysis (Obs/Calc) N; 7.34\% (7.40\%).

(b) from 7 : 9-Amino-2,4-diaza-1,5-dioxo-10-cyano-3-thio-2,4-diaryl-spiro[5.5]dec-9,10-diene (7, $0.005 \mathrm{~mol})$ was dissolved in acetic acid $(15 \mathrm{ml})$ and water $(2 \mathrm{ml})$. The reaction mixture was refluxed for 2 hours in an heating mantle. To this refluxing solution, o-phosphoric acid $(10 \mathrm{ml})$ was added dropwise in a span of 10 minutes. The reaction mixure was further refluxed for $24 \mathrm{hrs}$. It was then cooled and poured onto crushed ice, filtered, dried and crystallized from $50 \%$ ethanol. [yields of products by this route : $\mathbf{5 a}(73 \%) \& \mathbf{5 b}(81 \%)]$

\section{Acknowledgements}

The authors thank TIFR, Mumbai for mapping the spectra of their compounds.

\section{References and Notes}

1. T. Ooi, D. Ohara, K. Fukumoto, K. Maruoka, Org. Lett., 7, 3195 (2005).

2. M.C. Willis, J. Chauhan, W.G. Whittinham, Org. Biomol. Chem., 3, 3094 (2005).

3. Y. Chi, S.H. Gellman, Org. Lett., 7, 4253 (2005).

4. J. Comelles, M. Moreno-Manas, A. Vallribera, ARKIVOC, (ix), 207 (2005) and references therein.

5. B.C. Ranu, S. Banerjee, Org. Lett., 7, 3049 (2005).

6. M.S. Chande, Suryanarayan V, Tet. Lett., 43, 5173 (2002).

7. M.S. Chande, Khanwelkar R, Tet. Lett., 46, 7787 (2005).

8. M.S. Chande, Suryanarayan V, Chemistry of Heterocylic Compounds, 39(8), 1094 (2003).

9. M.S. Chande, Suryanarayan V, J Chem. Res, 6, 345 (2005).

10. I.N.D. Dass and S. Dutt, Proc Indian Acad.Of Science, 8A, 45 (1938).

Received on May 18, 2006. 